\title{
Juegos de estrategia y consecuencias inintencionadas: un modelo con resultados perversos de la crisis de la minería de Cartagena-La Unión
}

\section{Francisco Linares Martínez}

Universidad de La Laguna. Departamento de Sociología

flinares@ull.es

\section{Resumen}

Este artículo representa una aproximación al conflicto relativo a la continuidad de la actividad minera en la comarca de Cartagena-La Unión, que impulsó, en 1988, a la Sociedad Minera y Metalúrgica de Peñarroya a abandonar el distrito minero después de varias décadas de actividad. En primer lugar, brevemente, se da cuenta de las vicisitudes de la actividad minera a lo largo de la década de 1980, para llegar a estudiar los sucesos críticos del año 1988. En el análisis de estos acontecimientos se emplea una aproximación propia de la teoría de juegos para mostrar cómo fracasaron las negociaciones entre las partes implicadas y cómo del sistema de interacción emergieron consecuencias inintencionadas de signo perverso para algunas de ellas.

Palabras clave: acción colectiva, consecuencias inintencionadas, negociación, juegos de dos niveles, teoría de juegos.

Abstract. Games of strategy and unintended consequences: a model with perverse outcomes of the mining sector's crisis in Cartagena-La Unión

This paper represents an approximation to the conflict concerning the continuity of mining activity in the area of Cartagena-La Unión, which, in 1988, prompted Sociedad Minera y Metalúrgica de Peñarroya to leave this mine-district after several decades of activity. First of all it briefly accounts for the vicissitudes of mining activity throughout the eighties, coming to the study of the events happened in 1988. In the analysis of these events a game theoretic approach is used, in order to show how the bargaining among the parts broke down and how unintended and perverse outcomes for some of the parts emerged from the system of interaction.

Key words: collective action, unintended consequence, bargaining, two level games, game theory.

* Agradezco a los revisores anónimos de PAPERS sus aportaciones críticas a la última versión de este trabajo. También deseo mostrar mi agradecimiento a todas las personas que en su momento me brindaron su ayuda y su ánimo en el desarrollo de esta investigación. 


\section{Sumario}

Introducción

Dificultades en los años ochenta

El proyecto de Los Blancos III. Conflicto en torno a la continuidad de la minería

Juegos de estrategia en la comarca de Cartagena-La Unión

El desenlace del conflicto:

la venta de los activos de Peñarroya
Algunas características resaltables del proceso de interacción

Postescripto para curiosos: ¿cómo finalizó la crisis de la minería de la comarca de Cartagena-La Unión?

Bibliografía

\section{Introducción}

La minería ha acompañado a las gentes de la comarca de Cartagena desde que sus primeros pobladores se asentaran en la misma. Sin embargo, esta actividad sólo ha constituido un foco de desarrollo económico en breves periodos de su historia claramente acotados: durante la dominación romana, en la época republicana, principalmente entre la segunda mitad del siglo II aC y la primera mitad del siglo I aC (Martín Camino, 1996: 86); entre 1840 y 1930, periodo en el que la minería cartagenera conoció su máximo esplendor, y entre 1953 y 1991, periodo en el que la actividad cobró un nuevo auge merced a las nuevas técnicas de lavado del mineral por flotación diferencial y de explotación a cielo abierto ${ }^{1}$.

La protagonista indiscutible de esa última época es la multinacional de capital francés Sociedad Minera y Metalúrgica de Peñarroya-España (en adelante, Peñarroya), cuya producción, tras diversas dificultades, se inició en 1957, una vez desmontada la mina Emilia, y creció desde entonces de manera ininterrumpida hasta 1981. La estrategia de explotación de Peñarroya implicaba no sólo una inversión considerable en la constante ampliación y reconversión de sus medios de producción, sino también una incesante labor investigadora del potencial minero de la Sierra de Cartagena. De esta manera, a la altura de 1983, la sociedad minera explotaba simultáneamente seis cortas (pues a Emilia sucedieron, paulatinamente, Gloria, San Valentín, Los Blancos, San José y Tomasa); tenía en nómina a 555 empleados; era la primera productora de plomo en España y la tercera productora de zinc, aportando el $36,1 \%$ y el $17,4 \%$, respectivamente, del total nacional de estos metales (Gea Javaloy, 1986a: 274; 1986b: 324). Así mismo, se hallaba en estrecha relación con el tejido industrial cartagenero de entonces, pues suministraba inputs a tres de sus industrias: el 40\% del plomo consumido por la fundición Santa Lucia (propiedad de la misma empresa), el $60 \%$ del zinc consumido por la factoría de Zincasa, pos-

1. El conocimiento de la minería en la sierra de Cartagena-La Unión se debe fundamentalmente a las obras de los historiadores J.B. Vilar y P.M. Egea Bruno (1985, 1990). 
teriormente reconvertida en Española del Zinc, y el 80\% de la pirita de hierro consumida por Explosivos Río Tinto, posteriormente FESA (Gallego Rodríguez y Monllor Domínguez, 1993: 166-167). Sin embargo, este espectacular panorama se quebraría a lo largo de la década de 1980 (especialmente en la segunda mitad de la misma), teniendo lugar, a la altura de 1988, el abandono de la sierra minera por la compañía que le había dado un nuevo esplendor durante treinta años.

En el presente artículo se pretende dar cuenta de un conjunto de acontecimientos (fechados entre septiembre de 1987 y septiembre de 1988) que, cabe concebir, contribuyeron a este desenlace. Se huye de una aproximación estructuralmente determinista, en el sentido de que, si bien es un hecho cierto que la empresa minera pasaba por importantes dificultades en aquellos años (debido, entre otras razones, a la evolución a la baja del valor del mineral en los mercados internacionales), nuestra hipótesis es que el desenlace de los hechos se vio condicionado (en magnitud que presumimos importante, pero que, en cualquier caso, es imposible medir) por la compleja interacción entre los diversos agentes implicados en los acontecimientos: las élites políticas, los potenciales afectados (mineros y vecinos de Llano del $\mathrm{Beal}^{2}$ ) y la dirección de la empresa. Para dar cuenta de estos acontecimientos, en primer lugar, se exponen el cúmulo de circunstancias que daban a la minería cartagenera una condición de fragilidad. En segundo lugar, se analizan detalladamente los procesos de interacción estratégica entre las partes; este análisis se sostiene en la construcción e inspección de las características matrices de la teoría de juegos. En tercer lugar, se extraen las propiedades del sistema de interacción propuesto, prestando especial atención a la aparición de consecuencias inintencionadas. Finalmente, se plantea sucintamente la posibilidad de generalización del análisis para el estudio de otros casos empíricos.

\section{Dificultades en los años ochenta}

Como es conocido, la primera mitad de la década de 1980 fueron años de especial dificultad para la industria española, habida cuenta de que el gobierno central no podía demorar por más tiempo las medidas de reconversión que exigía el atajo de la crisis que sacudió a la economía mundial en la década de los setenta, y cuyos costes, en España, dada la delicada coyuntura política del momento, se había optado por no trasladar a los ciudadanos (Segura y otros, 1989). El estudio más detallado del comportamiento de este sector en la Región de Murcia durante los años de crisis es el de Fernández Valbuena (1989), que, empleando datos de los años 1978 a 1985, analiza la evolución de lo que él denomina «industria endógena» (transformación de productos agrarios, fabricación textil y de muebles), «industria exógena» (energía, industrias químicas y construcción naval), así como de un tercer subsector (que representa apro-

2. Ver más abajo. 
ximadamente un $25 \%$ del VAB y del empleo industrial) constituido principalmente por la minería, a su vez dominado hegemónicamente por Peñarroya. De los tres subsectores, aquél que manifiesta una peor evolución es precisamente el último, cuyo comportamiento es calificado por Fernández Valbuena como recesión destructora regresiva: "Lo que parece irreversible es el hundimiento del resto de la industria, dominado absolutamente por los sectores mineros, que [...] han experimentado reducciones tanto en el VAB como en el empleo y la productividad en el conjunto del periodo» (1989: 261).

En cuanto a la situación particular de Peñarroya, atendiendo a los datos de Gea Javaloy (1986a: 274, 1986b: 324), ésta permanecía en 1985 a la cabeza de los productores españoles de plomo, pero mientras que sus dos inmediatos competidores, Apirsa y Compañía de La Cruz, habían incrementado, entre 1983 y 1985, su participación en la producción nacional en 5,8 y 4,7 puntos porcentuales respectivamente, aquélla la había disminuido en 3,7 puntos. Respecto al sector del zinc, para el mismo periodo, Peñarroya es desplazada del tercer lugar por Apirsa, que incrementó su participación en la producción nacional en 5,5 puntos, mientras aquélla, ahondando la tendencia recesora de las otras grandes empresas (Asturiana del Zinc y Exminesa), disminuía su participación en 4,5 puntos. En el contexto de la Comunidad Autónoma de Murcia, las cifras no son mejores para la multinacional: su contribución a la producción minera regional pasa de representar el $73,8 \%$ en 1983 al 50,3\% en $1988^{3}$.

Para entender completamente la situación de la empresa en el año 1987, es preciso, además, prestar atención a algunos elementos determinantes o condicionantes (según el caso) de su actividad. Éstos son la «riqueza» de sus yacimientos, la evolución del valor de los metales, el grado de agotamiento de las explotaciones, la «cuestión ecológica» y la situación interna de la multinacional.

\section{Riqueza de los yacimientos}

Una de las características de los yacimientos de la Sierra de Cartagena es la baja ley ${ }^{4}$ de sus minerales, lo que permitía calificar a las explotaciones mineras en la misma como económicamente arriesgadas en ausencia de importantes inversiones en capital fijo. Así, Peñarroya explotaba yacimientos cuyo contenido en plomo fluctuaba alrededor del $1 \%$ del mineral. Como elemento de comparación, nótese que las leyes de los minerales explotados por sus más directos competidores españoles eran superiores: $1,7 \%$ en las minas de Aprisa y 1,5\% en las de Exminesa (Gea Javaloy, 1986a). Las leyes del mineral de zinc, si bien algo superiores, eran igualmente bajas, pues fluctuaban alrededor del $1,5 \%$.

3. Según datos de los anuarios estadísticos de la Región de Murcia.

4. La ley es la proporción de metal (por ejemplo, plomo) contenido en el mineral (en este caso, galena). Normalmente se expresa en tanto por ciento. 


\section{Cotizaciones de los metales en la Bolsa de Londres}

El valor de las producciones mineras depende de las cotizaciones de los metales en el mercado internacional. El precio del plomo venía entonces dado por una fórmula aplicada al valor que obtenía este metal en la Bolsa de Metales de Londres (London Metal Exchange), y el precio del zinc se obtenía a partir del Precio Productor Europeo, a su vez fuertemente influenciado por la cotización londinense (Gea Javaloy, 1986a: 277-279, 1986b: 327-329). Sin embargo, la evolución de estos mercados durante los años ochenta no fue especialmente favorable para el negocio minero. A la altura de 1986, escribía Gea Javaloy: «con los actuales niveles de precios, sólo resultaría económicamente viable la mitad de la producción de plomo occidental» (1986b: 330). Por aquel entonces, el precio del zinc era de 690 libras por tonelada métrica y el del plomo, de 258; nótese que la media de las cotizaciones de zinc y plomo durante la década de los setenta fueron de 773 y 583 libras de 1983 por tonelada, respectivamente.

\section{Cuestión ecológica}

Uno de los problemas de los intensivos métodos de explotación empleados por Peñarroya era la generación de un creciente volumen de estériles 5 . Rápidamente, el viejo sistema de verter el material estéril a las ramblas o de amontonarlo en "pantanos» quedaría obsoleto y, buscando una solución más satisfactoria, Peñarroya, avalada por un estudio de corrientes marinas, obtuvo de la Administración central en 1959 la autorización para verter sus estériles al mar en un punto que finalmente fue fijado en 250 metros de Punta de la Galera, extremo occidental de la bahía de Portmán (Vilar y Egea Bruno, 1990: 223). El mar no arrastró, tal y como estaba previsto, dichos estériles fuera de la bahía, sino que éstos se fueron sedimentando en su interior, lo que generó un problema ecológico de dimensiones aún no perfectamente evaluadas. El despertar de una importante sensibilización hacia las cuestiones ecológicas durante los años setenta y ochenta condujo a la Administración a presionar a la empresa minera para finalizar los vertidos al mar. El problema, sin embargo, no era fácil de resolver, pues exigía una inversión multimillonaria para la construcción de un nuevo lavadero. Esta inversión fue finalmente realizada por la sucesora de Peñarroya, Portmán Golf, en el año 1990.

\section{Agotamiento de los yacimientos}

La progresiva extinción de los recursos de las diversas cortas en explotación propició un estancamiento y posterior caída de la producción. La explota-

5. La actividad minera a cielo abierto produce dos tipos de estériles: el escombro fruto del desmonte y el material sobrante una vez lavado el mineral. Nos referimos aquí a este último tipo. 
Tabla 1. Reservas de mineral conocidas (toneladas).

\begin{tabular}{lcc}
\hline & Explotables & No explotables \\
\hline Emilia & - & 700.000 \\
Tomasa & 330.000 & 400.000 \\
San Valentín & 300.000 & - \\
Brunita & 780.000 & - \\
Los Blancos III & 17.700 .000 & 7.960 .000 \\
\hline TOTAL & 19.110 .000 & 9.060 .000 \\
\hline
\end{tabular}

Fuente: Peñarroya-España (1987b).

ción de la cantera Emilia finalizó en 1982; la de Los Blancos (I y II), en 1985, y la de Gloria se hallaba prácticamente finalizada en 1987. Así, en julio de 1987 las reservas explotables en las canteras históricas se cifraban en 1,4 millones de toneladas, es decir, poco más de seis meses de laboreo manteniendo el ritmo de explotación de 2,4 millones de toneladas al año. Las reservas comprobadas a finales de 1987 se hallan en la tabla 1. Como es fácil comprender, la continuidad de la actividad dependía directamente de la explotación de un nuevo yacimiento proyectado con el nombre de Los Blancos III.

\section{Reestructuración de la empresa}

Un factor cuya influencia, según varios indicios, no debe despreciarse pero del que, al mismo tiempo, se dispone de escasa información, es la fusión de la Sociedad Minera y Metalúrgica Peñarroya con la sociedad suizo-germana Preussag, para constituir el grupo multinacional Metaleurop, cuyos intereses se centraban en el negocio de fundición del metal. A raíz de esta fusión, consumada en abril de 1988, es muy posible que la dirección de París de Peñarroya perdiera interés por su rama de extracción minera, y la lucha mantenida por el cuadro directivo español para mantener las explotaciones de la Sierra de Cartagena finalmente no fuera un obstáculo para que éstas se pusieran a la venta.

\section{El proyecto de Los Blancos III. Conflicto en torno a la continuidad de la minería}

La Sociedad Minera y Metalúrgica de Peñarroya no se resignó ante el agotamiento de las reservas de sus canteras, de manera que en 1987 elaboró un proyecto para la explotación de una nueva corta, llamada Los Blancos III, que, cubriendo la cantera Sultana (en producción desde 1984), se extendía hasta el linde sureste de la pedanía cartagenera Llano del Beal, sita en la falda noreste de la sierra minera. Este proyecto contemplaba la explotación de 23,65 millones de toneladas de mineral (de las cuales 15,85 contenían un 0,81\% de plomo y un $2,37 \%$ de zinc, y las restantes 7,8 contenían un $0,41 \%$ de plomo y un 
$1,37 \%$ de zinc), lo que reportaría a la empresa unos beneficios netos cuya estimación oscilaba entre 2.500 y 8.600 millones de pesetas, dependiendo de la evolución de distintos parámetros económicos, en diez años ${ }^{6}$.

Dado que las labores mineras habrían de desarrollarse en un continuo acercamiento hacia Llano del Beal, la dirección de Peñarroya planeó ${ }^{7}$ una entrevista con los vecinos de esta pedanía para exponerles sus planes y negociar algún tipo de compensación por los posibles perjuicios. Atendiendo al relato ${ }^{8}$ de uno de los vecinos que asistieron a dicha reunión:

Nos llamó Peñarroya para enseñarnos los proyectos que tenía [...] Nos enseñaron una maqueta verde que era preciosa en la que se veía el pueblo y entonces, bueno, pues allí se veían unas rayas y en un determinado momento de la entrevista tiran de la maqueta, levantan una parte de la maqueta y se llevan medio pueblo. Y dicen: «bueno y esto nos vamos a llevar una parte del pueblo y dentro de unos años pues quedará, dentro de veinte años o diez años, quedará aquí, arriba, pues lo rellenaremos de tierra y quedará un parque cojonudo». Y nos quedamos sorprendidos, ¿no? O sea nos quedamos paralizados ante aquello. O sea era la desaparición del pueblo y la frase que dijeron fue: «bueno, la casa que tiremos, casa que pagamos». Nada más.

A los ojos de la dirección técnica de Peñarroya, los acontecimientos se desarrollaron de forma matizadamente distinta:

El proyecto contemplaba acercarse a los cuarenta metros famosos que decía la Ley de Minas ${ }^{9}[\ldots]$. Y ese proyecto llevaba una restauración en la zona tal [en las inmediaciones del pueblo], que instalaba un polideportivo..., bueno, un polideportivo, unas pistas deportivas, tal, una vez terminado, ¿no? Pero luego viene una segunda parte que dicen: «aunque realmente si se pudiera desarrollar el proyecto en su totalidad con las reservas que hay proyectadas, se llevaría esto». Entonces, la maqueta contemplaba destapar una parte que se iba más o menos al bar del Chupa [...]. O sea se llevaba un pellizco no muy grande, pero unas cuantas casas del pueblo. Entonces, eso, desgraciadamente de esa reunión lo que quedó fue el espíritu de que: «joder, han llegado allí, han hecho así y se han llevado las casas».

Sea como fuere, este proyecto desencadenó una fuerte contestación de los vecinos de Llano del Beal, quienes creían peligrar su identidad como pueblo si los planes de la sociedad minera se llevaban a efecto. Dicha contestación

6. Todos los datos se han extraído de Peñarroya-España, Proyecto de la corta Los Blancos III.

7. Nos referimos en este apartado a la dirección local de la empresa. Las diferencias entre los distintos niveles de dirección de la multinacional se abordan sucintamente al inicio del próximo apartado.

8. Cuando no se cite otra fuente, los fragmentos intercalados en el texto son extractos de entrevistas personales.

9. La Ley de Minas establecía que la distancia mínima entre una población y una explotación minera debía de ser de cuarenta metros. 
adoptó múltiples formas, desde las manifestaciones ante las correspondientes sedes institucionales hasta el bloqueo de la producción de la empresa concentrando personas en las pistas por las que debía de circular la maquinaria minera; acciones que tuvieron su eco en las administraciones local y regional, que se enfrentaban al difícil problema de reconciliar las demandas de los vecinos con los planes de una empresa que constituía uno de los pilares económicos de la comarca. El conflicto desatado apareció ante la opinión pública, a través de los diversos medios de comunicación, como sucesivas avalanchas de movilizaciones protagonizadas alternativamente por dos colectivos: los mineros de Peñarroya (que veían peligrar sus puestos de trabajo) y los vecinos de Llano del Beal. Paralelamente a éstas, tuvieron lugar una serie de reuniones bilaterales o multilaterales entre dichos colectivos y la Administración; reuniones cuyos acuerdos, a su vez, se convertían en la justificación de nuevas movilizaciones por parte del colectivo menos favorecido en cada caso.

La máxima tensión se alcanzó en los meses de enero y febrero de 1988, en los que vecinos y mineros protagonizaron sendos encierros exigiendo en cada caso a la Administración una actuación más favorable a sus intereses. En qué medida los acontecimientos de estos meses influyeron, o precipitaron, la decisión de la multinacional de vender sus activos mineros, no puede conocerse. De lo que sí existe evidencia es de que la empresa, como se ha mostrado, proyectó la explotación de los yacimientos con anterioridad a tales acontecimientos y manifestó su decisión de vender poco después de los mismos. De cualquier manera, el conflicto jamás fue resuelto, sino que se trasladó en septiembre de 1988 a la nueva propietaria de los activos mineros, la sociedad de origen autóctono Portmán Golf. Los sucesos de aquellos meses críticos (cuya cronología se expone en la tabla 2) pueden sintetizarse en los siguientes puntos:

1. Los vecinos inician (18 de enero) turnos de vigilancia de día y de noche, para evitar la posible realización de sondeos de prospección geológica requeridos por la Administración a la empresa para conocer el impacto de la explotación minera en el entorno del pueblo. Esta vigilancia se institucionalizará en un improvisado centro de reunión, bautizado como «la Cabaña», y se perpetúa hasta el año 1991.

2. Los representantes de las distintas administraciones se reúnen (8 de febrero) y acuerdan no aprobar el proyecto general de la cantera Los Blancos III, pero muestran la voluntad de aprobar el plan de labores ${ }^{10}$ de la sociedad minera para 1988 (que fijaba la línea de explotación a setecientos cincuenta metros del pueblo), condicionando su aprobación a los resultados de los pertinentes estudios técnicos.

3. Los mineros se encierran (17 de febrero) en la mina de San Rafael para exigir de la Administración una postura más explícita sobre el futuro de la minería en la región.

10. A las empresas mineras se les requiere la aprobación anual del correspondiente "plan de labores" para desarrollar su actividad. 
Tabla 2. Cronología de los acontecimientos de enero y febrero de 1988.

\begin{tabular}{|c|c|c|c|c|}
\hline Fecha & Vecinos & Administración & Mineros & Peñarroya \\
\hline 18 enero & $\begin{array}{l}\text { Inicio de los turnos } \\
\text { de vigilancia para } \\
\text { impedir la realización } \\
\text { de los sondeos. }\end{array}$ & & & $\begin{array}{l}\text { Suspensión } \\
\text { temporal de } \\
\text { los sondeos. }\end{array}$ \\
\hline 22 enero & \multicolumn{4}{|c|}{ Reunión de las partes a iniciativa del alcalde de Cartagena. } \\
\hline 30 enero & \multicolumn{4}{|c|}{$\begin{array}{l}\text { Manifestación en } \\
\text { La Unión. }\end{array}$} \\
\hline
\end{tabular}

\begin{tabular}{ccc}
\hline 4 febrero & $\begin{array}{c}\text { Bloqueo de la carretera } \\
\text { de acceso al lugar } \\
\text { donde se realizarían } \\
\text { los sondeos. }\end{array}$ & $\begin{array}{c}\text { Reunión de las } \\
\text { administraciones } \\
\text { locales y regional. }\end{array}$ \\
\end{tabular}

8 febrero Reunión de las partes para consensuar los acuerdos adoptados el día anterior:

- «No» (temporal) al proyecto de explotación Los Blancos III.

— «Sí» al plan de labores de 1988, que sitúa la línea de explotación a 750 metros de El Llano.

- Sometimiento de la explotación a los controles técnicos y legales oportunos.

\begin{tabular}{cl}
\hline 17 febrero & Encierro de trece \\
& mineros en la \\
& mina de San Rafael.
\end{tabular}

18 febrero Reunión en la que la Administración reitera su voluntad de que continúe la minería.

19 febrero Reunión en la que la Administración hace constar su voluntad de aprobar el proyecto de Los Blancos III, aceptando una distancia mínima desde El Llano de 175 metros.

20 febrero $\quad$ Fin del encierro.

22 febrero Encierro en el Ayuntamiento de Cartagena.

23 febrero

Reunión entre el alcalde de Cartagena y la

Administración Regional.

24 febrero Reunión en la que las partes reafirman su acuerdo en:

- Aprobar del plan de labores de 1988.

- Someter la explotación a los controles técnicos y legales pertinentes.

Fin del encierro.

Fuente: noticias del diario La Verdad. 
4. Los representantes del Gobierno regional se reúnen (19 de febrero) con la dirección local de Peñarroya y le muestran su voluntad de aprobar el proyecto de Los Blancos III, tras conocer los resultados de los pertinentes estudios, y aceptan, en su caso, la distancia de 175 metros al linde sur del pueblo como la mínima técnicamente necesaria para garantizar la rentabilidad de la explotación.

5. Los vecinos de Llano del Beal se encierran (22 de febrero) en el ayuntamiento de Cartagena y posteriormente transmiten al alcalde de la ciudad su intención de no aceptar los nuevos acuerdos entre la Administración y la empresa minera.

6. Los representantes del gobierno regional se reúnen ( 24 de febrero) con los representantes de los vecinos y se reafirman en los acuerdos a los que éstos se remitían (a saber, los alcanzados en la reunión del día ocho de febrero): aprobar el plan de labores de 1988, sometido a todos los controles técnicos y legales necesarios para garantizar el bienestar del colectivo afectado.

\section{Juegos de estrategia en la comarca de Cartagena-La Unión}

En esta sección se examina la interacción entre tres de los actores principales de los acontecimientos: los trabajadores de Peñarroya, los vecinos de Llano del Beal y el Gobierno regional. Como se verá, resulta evidente que existía una interacción estratégica entre estos actores. No resulta evidente, sin embargo que dicha relación estratégica existiera entre aquéllos y Peñarroya. En el caso de la multinacional, parece existir una disociación entre los intereses de los órganos directivos locales y nacionales (que atendieron diversas mesas negociadoras con la intención de superar las dificultades y continuar con la actividad) y el más alto nivel de dirección (París), principalmente preocupado por la política internacional de la empresa ${ }^{11}$. Más concretamente, cabe presuponer que, para la dirección de París, la cuestión de la actividad minera en Cartagena-La Unión se planteaba en términos de racionalidad paramétrica: con independencia de los intereses de la dirección local, la decisión de continuar o no se condicionaba a la evolución de circunstancias consideradas externas. Por tanto, no se incluye a la empresa como un actor relevante en el análisis de la dinámica del conflicto en términos estratégicos ${ }^{12}$.

La metodología empleada para analizar este proceso es la construcción y el escrutinio de las matrices características de la teoría de juegos. Esta aproximación implica asumir que los actores (o jugadores) son racionales, que saben que interactúan con otro actor racional, y que conocen sus preferencias y las pre-

11. Política a la que se ha hecho referencia anteriormente, en el subepígrafe titulado "Reestructuración de la empresa".

12. Por lo demás, la aportación de la dirección local a la solución del conflicto es la exigencia de continuar la explotación hasta la distancia de 175 metros del linde del pueblo, como única vía de mantener los puestos de trabajo, y el compromiso de adoptar las medidas que fueran necesarias en el desarrollo de su actividad para causar el menor daño posible a los vecinos. 
ferencias del actor con el que están interactuando ${ }^{13}$. El análisis se fundamenta en el concepto de equilibrio de Nash. Este equilibrio es el resultado que se alcanza como fruto de un proceso de mutua anticipación de las acciones que serán elegidas por cada jugador. Dicho proceso termina con la elección por cada jugador de su mejor respuesta a la acción (anticipada) del otro. Si se cumplen las premisas establecidas, las elecciones realizadas deben de confirmar las expectativas de los jugadores. Dado que el equilibrio es un conjunto de estrategias tal que ningún jugador puede obtener un resultado mejor cambiando unilateralmente su opción (puesto que su opción fue la mejor respuesta a la estrategia del otro jugador), se trata, por tanto, de un resultado estable.

\section{Interacción entre vecinos y mineros}

En la matriz 1 se presenta la interacción entre vecinos y mineros. En ella se representan las opciones de cada colectivo, que tiene que elegir entre movilizarse o permanecer pasivo. Cada combinación de acciones produce un resultado distinto, y cada resultado se ordena de mayor a menor, de manera que se asigna el valor arbitrario ${ }^{14}$ de 4 a la opción más preferida; el de 3 , a la segunda más preferida; el de 2, a la tercera más preferida, y el de 1 , a la menos preferida. El número anterior a las comas representa siempre la preferencia del jugador de las filas (en este caso, los trabajadores) y el posterior, la preferencia del jugador de las columnas (en este caso, los vecinos).

Recuérdese que las primeras protestas de los vecinos contra el acercamiento de la cantera Los Blancos III a su pueblo provocaron que los mineros acudiesen a los foros en los que se discutía la continuidad de la explotación minera y, posteriormente, emprendieran acciones más tenaces en defensa de sus

Matriz 1. Juego trabajadores-vecinos.

\begin{tabular}{|c|c|c|c|}
\hline & \multicolumn{2}{|c|}{ Vecinos } \\
\hline & & Movilización & Pasividad \\
\hline \multirow[t]{2}{*}{ Trabajadores } & Movilización & 2,2 & 4,1 \\
\hline & Pasividad & 1,4 & 3,3 \\
\hline
\end{tabular}

13. La construcción de este tipo de matrices no exige una medición cuantitativa del beneficio obtenido en cada caso por cada actor, sino tan solo especificar una serie de hipótesis sobre las preferencias ordinales (los ordenamientos de preferencias) de cada actor para cada posible resultado. Las hipótesis que en adelante se establecen se sostienen en la evidencia empírica fruto de entrevistas en profundidad y otras fuentes de documentación. Por otra parte, como argumenta Becker (1976), un requisito imprescindible (para evitar un razonamiento tautológico) es asumir que estas preferencias son fijas.

14. El valor numérico concreto asignado a cada resultado es irrelevante. Lo importante es el orden establecido entre los resultados. 
puestos de trabajo; por otra parte, las propias movilizaciones de los mineros dieron lugar a nuevas protestas de los vecinos. Parece, por tanto, que los intereses de cada actor y las expectativas sobre el comportamiento del otro pudieron ser el mecanismo que diera lugar a una dinámica conducente a una espiral de movilizaciones.

En los términos del entonces presidente del comité de empresa de PeñarroyaEspaña:

En este cúmulo de intereses, nosotros teníamos uno primordial, que era la defensa de los puestos de trabajo y ahí nos encontrábamos con que, por una parte, teníamos que enfrentarnos a lo que es un pueblo, como era el pueblo de El Llano, y teníamos que algunas veces hacer algo que [a] la empresa le convenía. Eran intereses creados. El interés de los trabajadores lógicamente, era el interés del mantenimiento de puestos de trabajo. El interés de la empresa era otro. Pero algunas veces convergían o se unían los dos intereses y eso era criticado por cierto sector de la sociedad, y principalmente del pueblo de El Llano, porque se criticaba a los representantes de los trabajadores, [...] porque no entendían que defendiéramos algo que iba en contra de sus intereses, pero nuestro propio interés sí que por lo menos prevalecía.

Y atendiendo al vicepresidente de la asociación de vecinos:

Siempre en el pueblo había..., ha habido siempre, es un pueblo trabajador, por supuesto, ha habido un respeto al trabajo. Lo que ocurre es que cuando perjudica seriamente los intereses personales de una persona pues entonces ahí hay otros principios de libertad o de derechos que están por encima de ese derecho [al trabajo] ¿no? Entonces en ese momento la asociación de vecinos o el conjunto del pueblo no estaba en contra de los puestos de trabajo pero sí estaba en contra de que abrieran esa cantera en las inmediaciones del pueblo, con las consecuencias que después determinaron, pero, vamos, que la postura nuestra era el oponernos a la cantera en las cercanías del pueblo.

Entendemos que no violentamos esta evidencia si asumimos que cada jugador prefiere:

- En primer lugar, ser el único en movilizarse, pues las movilizaciones de los vecinos son contraproducentes para los intereses de los mineros, y viceversa.

- En último lugar, ser el único en no movilizarse, pues entonces el otro jugador sería el único en negociar con la Administración.

Las hipótesis sobre los otros dos posibles resultados son irrelevantes para el resultado del análisis. Por conveniencia para la exposición asumimos, no obstante, que en segundo lugar ambos prefieren que nadie se movilice y, por tanto, en tercer lugar ambos prefieren que los dos se movilicen.

Con estas hipótesis, la estructura de la interacción entre vecinos y mineros es la del juego conocido como Dilema del Prisionero. Como es sabido, en 
este juego la conjunción de decisiones individualmente racionales produce un efecto socialmente ineficiente. Cada jugador tiene una estrategia estrictamente dominante (esto es, una estrategia que siempre es la mejor haga lo que haga el contrincante $)^{15}$, a saber, «movilización». Para comprobarlo, nótese que tanto en el caso de que los vecinos escogieran «movilización» como en el de que escogieran "pasividad», los trabajadores preferirían escoger «movilización» a "pasividad», puesto que en el primer caso se verifica que $2>1$, y en el segundo se verifica que $4>3$. Un razonamiento análogo explica la elección de los vecinos. De esta manera, el equilibrio de este juego (señalado con un asterisco) es que ambos colectivos se movilizan ${ }^{16}$.

\section{Interacción entre el Gobierno regional y los mineros}

En el juego representado en la matriz 2, los mineros pueden elegir, como en el caso anterior, entre "movilización» y "pasividad», mientras que el Gobierno regional elige entre no comprometerse a satisfacer las demandas de los trabajadores (opción que, por conveniencia de la exposición, se etiqueta como «vecinos») y comprometerse a satisfacer las demandas de los trabajadores (opción que, por conveniencia de la exposición, se etiqueta como "trabajadores») ${ }^{17}$.

Los intereses de los trabajadores en esta interacción pueden apreciarse, claramente, en la siguiente exposición del entonces secretario regional de minería de la UGT:

Nosotros lo que estábamos haciendo presión es para mantener la actividad minera [...]. No hacíamos ni siquiera mención de los vecinos, nunca. Ahora bien, nosotros cuando la Administración nos decía: «hombre, es que los veci-

Matriz 2. Juego gobierno regional-trabajadores.

\begin{tabular}{|c|c|c|c|}
\hline & \multicolumn{2}{|c|}{ Trabajadores } \\
\hline & & Movilización & Pasividad \\
\hline \multirow[t]{2}{*}{ Gobierno regional } & Vecinos & 1,2 & 3,1 \\
\hline & Trabajadores & 2,3 & 4,4 \\
\hline
\end{tabular}

15. Para una definición más rigurosa, consúltese cualquier manual de teoría de juegos; por ejemplo, Gibbons (1993).

16. Alterando las relaciones de orden sobre la segunda y la tercera preferencias, no se cambiaría la conclusión de que movilización es una estrategia dominante, pero sí la de que el resultado de este juego es subóptimo. Ello, no obstante, es secundario para el análisis conjunto de todas las interacciones, como se verá más adelante.

17. Dicha conveniencia, por lo demás, refleja el carácter de «suma cero» que, a los ojos del espectador y probablemente de algunos protagonistas, parecía mostrar el conflicto. 
nos dicen que se les caen no sé qué, que se le cae...», mire usted, vamos a reunirnos, a ver si es verdad, vamos a buscar soluciones, y yo entiendo que si nosotros, desde el principio, los vecinos demuestran que se sienten tal, pues tendremos que reconocer nosotros mismos que no podemos continuar, pero si eso no es así, nosotros lo que exigimos a ustedes es que le hagan ver a los vecinos que hay que continuar, porque de ahí está la supervivencia de más de mil quinientas personas.

Por otra parte, las preferencias del Gobierno regional se desprenden de las palabras del entonces presidente de la Región de Murcia:

Allí estábamos todos a favor de resolver el problema. Y estábamos en un dilema, evidentemente. Porque, por un lado, [...] estaban los trabajadores, la empresa con su... Yo ya me hago abstracción de la..., como muchas veces hacíamos abstracción, de la legalidad que amparaba a la empresa. A nosotros nos preocupaban los quinientos trabajadores. ¿Sabe el potencial explosivo que suponía la liquidación de quinientos trabajadores en una zona ya deprimida [como La Unión]? Entonces esto nos angustiaba, por un lado. Por otro lado, también nos angustiaba el tinglado que tenían montado los del Llano del Beal, con su caseta aquella... «la cabaña».

En concreto, asumiremos que el Gobierno regional prefiere:

- En primer lugar, comprometerse a satisfacer las demandas de los trabajadores y que éstos no se movilicen.

- En último lugar, no comprometerse a satisfacer las demandas de los trabajadores y que éstos se movilicen.

Las hipótesis sobre las dos opciones intermedias, como en el caso anterior, son irrelevantes para el resultado final del análisis, aunque asumiremos que prefiere la opción en la que los trabajadores no se movilizan (y no existe compromiso) antes que la opción en la que sí se movilizan.

Respecto a los trabajadores, asumimos que prefieren:

- En primer lugar, que el Gobierno regional se comprometa a satisfacer sus demandas y no (tener que) movilizarse.

- En último lugar, que el Gobierno regional no se comprometa a satisfacer sus demandas y permanecer pasivos.

En este caso, las opciones segunda y tercera sí son relevantes para el resultado del análisis; no obstante, las preferencias son obvias:

- En segundo lugar, que el gobierno regional se comprometa a satisfacer sus demandas y movilizarse.

- En tercer lugar, que el gobierno regional no se comprometa a satisfacer sus demandas y movilizarse.

Así, las preferencias del gobierno regional muestran un mayor interés por satisfacer la exigencia de garantizar los puestos de trabajo que por las reivin- 
dicaciones de los vecinos. De esta forma, «trabajadores» es la estrategia dominante del Gobierno regional en este juego. En el caso de los mineros, no existe una estrategia dominante, sino que éstos prefieren «movilización» en el caso de que aquél elija "vecinos» (puesto que $2>1$ ), y "pasividad» en el caso de que elija "trabajadores» (puesto que $4>3$ ). En consecuencia, el juego tiene un único equilibrio en el que el Gobierno regional elige comprometerse a satisfacer las demandas de los trabajadores y éstos eligen no movilizarse, con lo que ambos jugadores obtienen el mejor resultado posible ${ }^{18}$.

\section{Interacción entre el Gobierno regional y los vecinos}

En el juego representado en la matriz 3 los vecinos tienen la oportunidad de escoger entre «movilización» y "pasividad». El Gobierno regional en esta ocasión debe de escoger entre comprometerse a satisfacer las demandas de los vecinos (que, por conveniencia, etiquetamos "vecinos») o no comprometerse a satisfacer las demandas de los vecinos (que, por conveniencia, etiquetamos «trabajadores») ${ }^{19}$.

La situación se plantea desde el punto de vista del Gobierno regional, atendiendo a las palabras de su presidente, del siguiente modo:

[Era] una posición muy incómoda la de la Administración. Y al final ¿qué es lo que busca la Administración? El diálogo. Vamos a tratar de llegar a un acuerdo de que la empresa pueda trabajar, de que se le exija a la empresa determinadas

Matriz 3. Juego gobierno regional-vecinos.

\begin{tabular}{|l|l|c|c|}
\hline \multicolumn{2}{|c|}{} & \multicolumn{2}{c|}{ Vecinos } \\
\cline { 3 - 4 } Gobierno regional & Vecinos & $\mathbf{2 , 3}$ & Pavilización \\
\cline { 2 - 4 } & Trabajadores & $\mathbf{1 , 2}$ & $\mathbf{4 , 1}$ \\
\hline
\end{tabular}

18. Se podría sugerir un ordenamiento de preferencias alternativo en el que las dos primeras preferencias de los trabajadores se mantienen y las dos últimas se invierten, en comparación con el ranking propuesto. Esta alteración no produciría ningún cambio en el resultado del análisis del juego de la matriz 2 (el equilibrio seguiría siendo el mismo), pero sí alteraría la lógica global del análisis propuesto en el conjunto del artículo. Una mente suspicaz podría argumentar que los números se han escogido de tal forma que se produzca un resultado premeditado. Lo que el autor defiende, sin embargo, es que los ordenamientos de preferencias propuestos «tienen sentido» desde el punto de vista de los motivos de los actores y, además, responden a la evidencia empírica. No sólo parece difícil argumentar con sentido que "pasividad» era la estrategia dominante de los trabajadores (y, en el caso de la matriz 3 - más adelante- de los vecinos), sino que además los hechos desmienten dicha hipótesis.

19. Nótese que las etiquetas de las estrategias del Gobierno regional en las matrices 2 y 3 no significan exactamente lo mismo. 
condiciones de ese trabajo, cómo tiene que hacer ese trabajo, y por tanto que se mantengan los puestos de trabajo, y luego vamos a ver de molestar lo mínimo a estos señores de Llano del Beal que tienen razón [en] que se les molesta, ¿no?

Mientras que para los vecinos la situación es bien distinta, ya que, según uno de los vocales de su asociación:

La sensación más bien era de estar desasistidos [...]. O sea, decir, no tenemos un ayuntamiento que sea nuestro ayuntamiento, no tenemos una administración que sea nuestra administración. Vemos a la Administración al servicio de la empresa, descaradamente. Descaradamente firmaban pactos entre la empresa y la Administración sin contar para nada con nosotros, que además eran absolutamente irrealizables, se intentaba buscar cancamanganas y caminos legales para llevarnos al huerto, se intentaba desacreditarnos por vía de medios de comunicación en vez de buscar puntos de contacto y puntos de conexión, cuando además lo más triste es que nosotros teníamos la voluntad de diálogo, y una voluntad manifiesta y expresa.

Asumimos que el Gobierno regional prefiere:

- En primer lugar, que los vecinos no se movilicen y no comprometerse a satisfacer sus demandas.

- En último lugar, que los vecinos se movilicen y no comprometerse a satisfacer sus demandas.

Las preferencias intermedias, una vez más, son irrelevantes para el resultado del análisis, pero, en cualquier caso, asumiremos que prefiere, en segundo lugar, que los vecinos no se movilicen y comprometerse a satisfacer sus demandas y, en tercer lugar, que los vecinos sí se movilicen a pesar de que se compromete a satisfacer sus demandas

Entendemos que estas cuatro hipótesis reflejan la evidencia de que: primero, el Gobierno regional se hallaba más preocupado por el problema de los trabajadores que por el de los vecinos; segundo, no obstante, también era sensible al problema de los vecinos; tercero, la preocupación por ambos problemas se hallaba sometida a una tensión interna, puesto que, llevadas hasta sus últimas consecuencias, las demandas de ambos colectivos encerraban un carácter de suma cero: o se explota la cantera (y se molesta a los vecinos en alguna medida) o se deja de explotar la cantera (y se despide a los trabajadores $)^{20}$.

20. Las hipótesis en las que se sostiene este ordenamiento de preferencias son especialmente importantes, porque de ellas depende el resultado global del análisis. Un ordenamiento en el que se asignara el máximo valor a la opción «vecinos-pasividad» (VP) sería incoherente con la hipótesis, sostenida en el epígrafe anterior, de que para el Gobierno regional era más importante el problema del mantenimiento de los puestos de trabajo que las reivindicaciones de los vecinos. Por tanto, el valor más alto debe de asignarse a la opción «trabajado- 
Con respecto a los vecinos, asumimos que tiene un ordenamiento análogo al de los trabajadores ${ }^{21}$, ya que se prefiere:

- En primer lugar, que el Gobierno regional se comprometa a satisfacer sus demandas y no movilizarse.

- En segundo lugar, que el Gobierno regional se comprometa a satisfacer sus demandas y movilizarse.

- En tercer lugar, que el Gobierno regional no se comprometa a satisfacer sus demandas y movilizarse.

- En cuarto lugar, que el Gobierno regional no se comprometa a satisfacer sus demandas y no movilizarse.

El juego entre el Gobierno regional y los vecinos ofrece una dificultad no presente en los anteriores: no existe ningún par de acciones que sean mutuas mejores respuestas y, por tanto, determinen un equilibrio estable. Si el Gobierno regional decide no comprometerse, los vecinos prefieren movilizarse (porque $2>1$ ), y si el Gobierno regional decide comprometerse, los vecinos eligen no movilizarse (porque $(4>3)$; en este último caso, sin embargo, el gobierno regional prefiere no comprometerse antes que comprometerse (porque $4>3$ ). Es decir, en el caso del Gobierno regional, la estrategia «vecinos» se prefiere a «trabajadores» si, y sólo si, los vecinos eligen «movilización»; pero éstos prefieren la estrategia «movilización» a "pasividad» si, y sólo si, aquél elige «trabajadores».

Ello no significa que el juego no tenga un equilibrio, sino que para cada uno de los jugadores la opción óptima no es elegir siempre la misma acción, sino elegir cada acción con frecuencias distintas. La distribución de frecuencias relativas asignadas a cada acción se denomina «estrategia mixta». Un conjunto de distribuciones de frecuencia, una para cada jugador, tal que cada una es la mejor respuesta a la distribución de frecuencias elegida por el otro jugador, es un equilibrio en estrategias mixtas. Ello implica que la estrategia mixta de equilibrio de cada jugador sea la distribución de probabilidad que hace que el otro jugador sea indiferente entre sus dos opciones ${ }^{22}$.

En el caso de la matriz 3, asignamos la incógnita $q$ a la probabilidad con la que el Gobierno regional escogerá «vecinos»; $(1-q)$ es, por tanto, la pro-

res-pasividad“ (TP). La segunda preferencia, sin embargo, debe de ser VP, ya que, si fuera "trabajadores-movilización» (TM), el Gobierno regional tendría una estrategia dominante: elegir trabajadores, hicieran lo que hicieran los vecinos. Ello implicaría una absoluta falta de sensibilidad hacia las reivindicaciones de los vecinos, lo que es contradictorio con la evidencia empírica. En cuanto a las dos últimas preferencias, si asumimos que el gobierno regional prefiere TM a VM, entonces de nuevo la estrategia "trabajadores» sería dominante, y el juego construido con las preferencias de los vecinos (explicitadas más adelante) terminaría con el equilibrio, en este caso subóptimo, TM.

21. Se aplican aquí, por tanto, argumentos similares a los expuestos en la nota número 16, referida al ordenamiento de preferencias de los trabajadores.

22. Una exposición más precisa puede hallarse en cualquier manual introductorio a la teoría de juegos. Véase, por ejemplo, Gibbons (1993). 
babilidad con la que escogerá «trabajadores». Y asignamos la incógnita $r$ a la probabilidad con la que los vecinos escogerán «movilización»; $(1-r)$ es, por tanto, la probabilidad con la que escogerán «pasividad». En equilibrio, el Gobierno regional escogerá «vecinos» con una probabilidad $q^{*}$, y los vecinos escogerán "movilización» con una probabilidad $r^{*}$. Aunque el espacio de este artículo nos impide analizar el juego con más detalle, es fácil demostrar que $q^{*}$ será tanto menor cuanto mayor sea la diferencia entre los valores asignados a lo que los vecinos ganen eligiendo "pasividad" y eligiendo «movilización», cuando el Gobierno regional elige «vecinos». Y que $r^{*}$ será tanto menor cuanto mayor sea la diferencia entre los valores asignados a lo que el Gobierno regional gane eligiendo "vecinos» y eligiendo "trabajadores», cuando los vecinos eligen «movilización». En cualquier caso, sea el que fuere el valor de estas incógnitas, este equilibrio siempre exige algún grado de movilización por parte de los vecinos y algún grado de compromiso para satisfacer sus demandas por parte del Gobierno regional.

\section{Interacción a tres bandas}

En resumen, por una parte, en el caso del juego entre el Gobierno regional y los mineros las estrategias de equilibrio son «trabajadores» (es decir, comprometerse a satisfacer las demandas de los mineros) y «pasividad». Por otra parte, sin embargo, el par de estrategias mutuamente óptimas del juego entre aquél y los vecinos implican algún grado de movilización por parte de éstos $y$ algún grado de compromiso con éstos por parte de aquél. Esto significa que, en tanto en cuanto el Gobierno regional no puede eludir jugar ambos juegos, le es imposible tomar una decisión que sea globalmente óptima.

Así, si elige su estrategia de equilibrio del juego con los trabajadores, entonces para los vecinos será óptimo elegir una probabilidad de movilización $r>r^{*}>0$. Pero al mismo tiempo si el Gobierno regional elige $q^{*}$, con el fin de mantener $r=r^{*}$, entonces se desvía de su equilibrio del juego con los trabajadores (puesto que $q^{*}>0$ ), y se desplaza en la matriz 2 de la celda («trabajadores», "pasividad»), con pagos (4,4), a la celda («vecinos», "pasividad»), con pagos $(3,1)$. Esto, a su vez, produciría un cambio en la elección de los mineros de «pasividad» a «movilización», ya que, cuando el Gobierno regional elige «vecinos» (es decir, no comprometerse a satisfacer las demandas de los mineros), la segunda estrategia les reporta una utilidad mayor $(2>1)$. En esta situación, el Gobierno regional se hallaría en el peor de los casos posibles $(1<3)$, por lo que tendría que volver a escoger «trabajadores» (a lo cual los mineros reaccionarían volviendo a la posición inicial de equilibrio). Sin embargo, esta última elección desencadenaría una nueva escalada de movilizaciones por parte de los vecinos, con lo cual volvería a reproducirse el ciclo descrito. Por lo tanto, debe concluirse que el problema planteado en los eclécticos términos elegidos por el Gobierno regional era irresoluble.

La dinámica del conflicto, representada en el diagrama 1, puede sintetizarse en los siguientes puntos: 


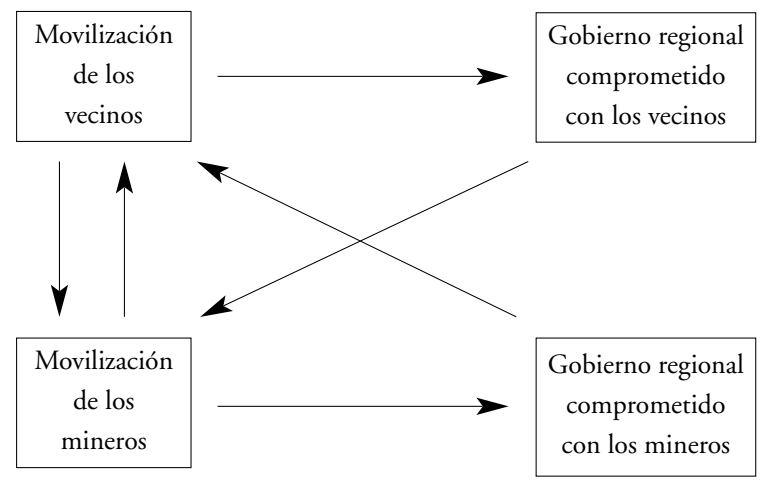

Diagrama 1. Dinámica interactiva.

- Un proceso de retroalimentación entre las movilizaciones de los vecinos y las movilizaciones de los mineros.

- La movilización de cada colectivo producía, así mismo, una respuesta del Gobierno regional en que mostraba su sensibilidad hacia los intereses del colectivo.

- El compromiso del Gobierno regional con una de las partes retroalimentaba la movilización de la otra parte.

Este modelo del conflicto resulta coherente con la evidencia empírica mostrada sobre los acontecimientos de enero y febrero de 1988:

1. Los vecinos de Llano del Beal siempre se hallaban en algún grado, variable, de movilización, como muestra el hecho de que establecieran turnos de vigilancia que se sucedieron de manera ininterrumpida desde comienzos de enero de 1988.

2. El Gobierno regional se mostró sensible desde el comienzo a las demandas de los vecinos y exigió a la empresa minera un mayor control de la seguridad de sus labores, así como un estudio de mecánica de rocas y vibraciones para conocer su posible impacto sobre el pueblo.

3. Los trabajadores comenzaron sus movilizaciones tan pronto percibieron que las demandas de los vecinos podían poner en peligro la continuidad de sus puestos de trabajo.

4. El Gobierno regional también se mostró especialmente sensible a las primeras movilizaciones de los mineros, como muestra su pronta respuesta al encierro de éstos en la mina de San Rafael, iniciado el día diecisiete de febrero y finalizado cuatro días más tarde, tras un acuerdo entre el comité de empresa y la propia Administración.

5. Los acuerdos de la Administración con los trabajadores, a su vez, desencadenaron mayores movilizaciones de los vecinos; en concreto, el encierro en el Ayuntamiento de Cartagena, el día veintidós del mismo mes. 


\section{El desenlace del conflicto: la venta de los activos de Peñarroya}

Una vez transcurridos los acontecimientos de enero y febrero de 1988, el Consejero de Economía e Industria defendía las gestiones de su departamento ante la Comisión de Política Sectorial de la Asamblea Regional de Murcia en los siguientes términos:

Hemos llegado a un, digamos, preacuerdo con la empresa que era quizá el elemento más difícil de todo esto y me da la impresión de que, de cara a la opinión pública, pues se ha infravalorado la importancia, por así decir, de la reunión con la empresa en la que de un planteamiento inicial de llegar a dentro del pueblo a que en la actualidad ha firmado un documento en el que se compromete a garantizar los puestos de trabajo y la explotación viable económicamente, fijando como mínimo una distancia de ciento setenta y cinco metros.

[...] Yo tengo el pleno convencimiento de que sí van a acometer la explotación de este mineral, salvo que sucedan otras cosas, que esto siempre es futurible y que cualquiera sabe qué es lo que puede ocurrir ${ }^{23}$.

Aproximadamente un mes y medio más tarde de los acontecimientos descritos, el catorce de abril de 1988, se sometió a votación en la Asamblea Regional el dictamen de la Comisión de Política Sectorial en relación con la apertura de la cantera Los Blancos III, elaborado por los diputados miembros de dicha comisión a petición de escrito de catorce de octubre de 1987 de la asociación de los vecinos de Llano del Beal. En dicho dictamen se lee:

De las comparecencias de todas las partes implicadas, y con los datos aportados, se ha podido deducir la conveniencia de la explotación de la cantera Los Blancos III para el mantenimiento de los puestos de trabajo de PeñarroyaEspaña, S. A., pero haciéndolo en las mejores condiciones posibles para salvaguardar los intereses de los habitantes de El Llano del Beal, en cuanto a la mejora de su calidad de vida y la seguridad de su pueblo ${ }^{24}$.

La aprobación de este dictamen por la mayoría absoluta de los diputados del parlamento regional produjo una violenta reacción por parte de los vecinos, que esperaban en las puertas de la Asamblea Regional ${ }^{25}$ y que, una vez en Llano del Beal, decidieron cortar el acceso a la cantera, a fin de impedir el desarrollo de las labores mineras. La reacción de la dirección de Peñarroya ante esta nueva parada de la producción se recoge en las siguientes declaraciones del entonces presidente de su comité de empresa:

23. Comparecencia del Sr. consejero de Economía e Industria ante la Comisión de Política Sectorial de la Asamblea Regional de Murcia, 21/3/1988, p. 93 y s.

24. Dictamen de la Comisión de Política Sectorial, en relación con la apertura de la cantera Blancos III, en el Llano del Beal, por la Sociedad Minero-Metalúrgica Peñarroya-España, S.A., Boletín Oficial de la Asamblea Regional de Murcia, II Legislatura, n.o 45, 20/4/1988, p. $1232-1234$.

25. La Verdad, 15/4/1988. 
Me acuerdo que me llaman ese día a las seis de la mañana a casa, el secretario del comité [...], en el que había un problema de que Peñarroya decía que cerraba. Entonces tenemos una reunión urgente, en las propias dependencias de la empresa, en que nos dice la dirección que los vecinos habían cortado el acceso a Los Blancos III, no podíamos trabajar, con lo cual esa situación ya no se podía mantener, y que ellos presentaban un cierre patronal.

En este escenario los trabajadores decidieron cortar con sus máquinas diversas carreteras regionales y nacionales, lo que produjo una situación de caos tal que el entonces Delegado del Gobierno en Murcia optó por enviar efectivos de la Guardia Civil para desalojar a los vecinos de las pistas mineras, eliminando así el obstáculo que impedía que la empresa continuara con su producción. No obstante, quince días más tarde, el tres de mayo de 1988, el nuevo director general de Peñarroya-España anunciaba al comité de empresa la decisión de vender la explotación: «hay que salvar lo salvable y silicatos no es salvable» ${ }^{26}$.

\section{Algunas características resaltables del proceso de interacción}

\section{Desconfianza de las partes en conflicto hacia el mediador}

Un sobresaliente rasgo común de las correspondientes definiciones de la realidad de las partes en litigio - trabajadores y vecinos - es la desconfianza hacia el mediador. Efectivamente, en cada uno de los colectivos es posible percibir una arraigada sospecha de que el mediador se halla al servicio de la otra parte. Así se desprende, por ejemplo, del relato del entonces secretario regional de minería de UGT:

Nuestras reivindicaciones, nuestras presiones, terminaban siempre en una reunión con la Administración. Y [en] esas reuniones con la Administración, siempre ellos manifestaban de que la mina pues tenía que continuar, lo único que pasa es que nunca han sido capaces de hacerle entender o hacerle ver a los vecinos de que la mejor solución era esa. Y me da la impresión que había políticos que decían a los vecinos: «no preocuparos que vamos a decir a los mineros que siguen pero vosotros ya sabéis que, diga lo que diga la prensa, no va a seguir».

Impresión muy similar a la recogida en la siguiente declaración de uno de los vocales de la asociación de vecinos:

Nosotros con la Administración teníamos una gran desconfianza, con toda la Administración. Con la Administración, con el Gobierno regional, o sea, con todo el mundo. Sabíamos que no teníamos..., nosotros los considerábamos que no eran..., eran nuestros enemigos, o sea que estaban enfrente de nosotros. O sea que no teníamos de la Administración la defensa que creíamos que teníamos que tener [...]. O sea que ellos pertenecían... estaban cerca de la empresa y nosotros pues, bueno, éramos los que estábamos protestando. 
Cualesquiera que fueran las fuentes endógenas de la desconfianza de los colectivos, lo que interesa resaltar es que ésta tiene también una fuente exógena, a saber, la propia estructura del conflicto, cuyo efecto multiplicador de las movilizaciones de unos y otros no sólo tiene la consecuencia de elevar el grado de tensión, sino que, lo que resulta de especial trascendencia para el punto que nos ocupa, obliga a la Administración regional a posicionarse públicamente sobre las exigencias del colectivo que en cada momento es protagonista de las movilizaciones, de manera tal que, al sucederse éstas periódicamente, se genera a los ojos de ambos una imagen contradictoria de los intereses defendidos por la misma Administración.

Esta sensación de traición acompañó a los vecinos de Llano del Beal durante toda la crisis. Ni que decir tiene que si la posibilidad de que se hallara una solución al conflicto dependía de la desmovilización de los vecinos de Llano del Beal, y esto a su vez dependía de una negociación exitosa entre los mismos y el Gobierno regional ${ }^{27}$, no es difícil comprender que la desconfianza de los vecinos hacia las intenciones de aquél lastraba las posibilidades de entendimiento. En este sentido, resulta ilustrativo el siguiente extracto del diario de uno de los vecinos del pueblo (Urrea, 1992), posteriormente publicado por la propia asociación:

Muchas familias hacen preparativos para pasar esta noche tan significativa en la Cabaña, el recuerdo y la obligación no se olvida, hasta que haya algún gobernante que tenga dignidad y confirme que la cantera Los Blancos III está completamente anulada. $(24 / 12 / 1988)$

\section{Consecuencias no intencionadas}

Atendiendo al análisis anterior, ¿qué consecuencias se derivan de la movilización emprendida por cada uno de los colectivos para defender sus intereses? En primer lugar, cada colectivo, como ya se ha repetido, contribuía con su movilización a una espiral de tensión. En segundo lugar, esta dinámica, que suscitaba continuas reacciones de la Administración, producía también (como se acaba de mostrar) una gran sospecha hacia los intereses del mediador por parte de ambos, lo que hacía más difícil llegar a un acuerdo. Cada movilización, finalmente, tenía el efecto de "atar las manos» de la Administración en su negociación con el otro colectivo, entorpeciendo así la solución del conflicto a través de cauces negociadores. En definitiva, cada colectivo emprendiendo acciones reivindicativas para la defensa de sus intereses, generaba efectos contraproducentes para sus propios intereses.

Esta conclusión tiene especial relevancia en el caso de los mineros. Sin duda, no es posible conocer qué es lo que hubiera ocurrido si los trabajadores no se hubieran movilizado; pero sí parece posible conjeturar que, hasta donde los argumentos esbozados anteriormente sean plausibles, su movilización con-

27. Es imprescindible recalcar el carácter meramente hipotético de estas afirmaciones. 
tribuyó a generar una situación en la que ciertamente se ponía en entredicho la viabilidad de sus puestos de trabajo. En la medida en que ello fue así, el resultado de la movilización de los trabajadores es ciertamente paradójico. Asistimos, por tanto, a la emergencia de una consecuencia inintencionada de un signo perverso con relación a sus intereses, así como a los de la Administración (en tanto en cuanto, para ella, el principal problema a resolver fuera el de los puestos de trabajo): a diferencia de las consecuencias inintencionadas producto de la «mano invisible» de A. Smith (mecanismo por el que, como es sabido, la ciega persecución de los propios intereses tiene un efecto redundante sobre la consecución de los intereses de los demás), en este caso parece operar una "mano negra» que hace que la ciega persecución de los propios intereses tenga, efectivamente, un efecto positivo sobre los intereses de los demás (en este caso, los vecinos), pero a expensas de un inesperado sacrificio de los intereses propios.

\section{Juegos de dos niveles}

En su análisis de Lógica y sociedad, J. Elster (1994) traza una sutil distinción entre los conceptos de contrafinalidad y suboptimalidad ${ }^{28}$. Ambos fenómenos presuponen (como en nuestro análisis) actores racionales; y ambos fenómenos tienen el mismo efecto: producen consecuencias contraproducentes para los actores. Pero en cada caso esa consecuencia tiene un origen distinto: en el primer caso, los actores desconocen las características del juego, mientras que en el segundo el conocimiento de las mismas conduce a un efecto perverso. Tomando como ejemplo el Dilema del Prisionero (matriz 1), podría ejemplificarse la distinción diciendo que el resultado contraproducente (la espiral de movilizaciones) es un caso de contrafinalidad si los colectivos deciden movilizarse sin ser conscientes de la verdadera naturaleza del conflicto (es decir, pensando que serán los únicos en movilizarse), y se trata de un caso de suboptimalidad si los colectivos deciden movilizarse a pesar de ser conscientes de la verdadera naturaleza del conflicto (es decir, para no ser los únicos en no movilizarse).

No creemos, sin embargo, que las consecuencias perversas del conflicto se correspondan ni con un caso de contrafinalidad ni con un caso de suboptimalidad. La razón es que en nuestro análisis presuponemos, por una parte, que los actores conocen el juego al que estaban jugando en cada momento, pero, por otra parte, juegan distintos juegos con distintos actores. Es decir, el resultado final del proceso no es el equilibrio de Nash de un juego de tres jugadores, sino el fruto de un complejo sistema de interacciones en el que se con-

28. Existen diversas clasificaciones de consecuencias inintencionadas. Algunas de estas clasificaciones constituyen una tipología (quasi)exhaustiva, pero no tienen valor explicativo (e. g. Baert, 1991). Otras no pretenden ser exhaustivas y hacen énfasis en los elementos explicativos (e. g. Merton, 1936). Nuestras propias reflexiones pueden hallarse en Linares (2003). 
catenan cíclicamente diversos puntos de equilibrio. Esta aproximación más compleja puede abordarse analíticamente empleando el concepto de «juego de dos niveles» desarrollado por R. Putnam (1988).

Haciendo una analogía con el análisis de Putnam ${ }^{29}$, un mediador (en nuestro caso, el Gobierno regional) desarrolla una doble negociación, una con cada parte. Se define como "conjunto ganador» (win set), el conjunto de posibles acuerdos que el mediador puede alcanzar con una de las partes y que, además, serían aprobados también por la otra parte. En el caso que nos atañe, podemos denominar $\mathrm{V}$ al conjunto de acuerdos posibles con los vecinos que serían aprobados (también) por los trabajadores, y podemos denominar T al conjunto de acuerdos posibles con los trabajadores que serían aprobados (también) por los vecinos. Un acuerdo global requiere que $\mathrm{V}$ y $\mathrm{T}$ se solapen en alguna medida. Por tanto, cuanto mayores sean V y T mayores serán las posibilidades de un acuerdo. Ahora bien, paradójicamente, un «conjunto ganador» pequeño de una de las partes podría dar ventaja a esa parte, pues el mediador, en la otra mesa negociadora, tendría que argumentar que tiene "las manos atadas». Al mismo tiempo, a nadie se le escapa que si V y T son demasiado pequeños probablemente no se solapen nunca: el Gobierno regional tendrá ambas manos atadas y nunca se producirá el acuerdo global.

Así, por ejemplo, tras conocer el resultado de la reunión del diecinueve de febrero de 1988, bajo la presión del encierro de los mineros, entre la empresa y la Administración (en la que ésta última manifiesta la voluntad de aprobar el proyecto de Los Blancos III fijando la distancia mínima de 175 metros al pueblo), los vecinos, en un comunicado firmado por el presidente de su asociación, muestran su desconcierto ante «la fijación con carácter previo a los estudios y a los pronunciamientos legales, la fijación de una distancia arbitraria que sólo depende de la situación del filón de mineral», concluyendo: «nos manifestamos en contra de dicho acuerdo que contradice la voluntad que la Administración expuso en la reunión de las partes implicadas el ocho de febrero pasado» ${ }^{30}$.

Esta situación, sin duda, podría ser concebida de manera simplificada como un único juego de tres jugadores con un equilibrio subóptimo (al menos para dos de ellos); esto es, como si los tres jugadores se sentaran simultáneamente en una única mesa de negociación. En tal caso, las consecuencias inintencionadas constituirían un ejemplo de suboptimalidad (como en el Dilema del Prisionero), y serían fácilmente predecibles, bajo la presunción propia de la teoría de juegos de que existe un "conocimiento común» de las características de la interacción, de tal forma que cada jugador es capaz de anticipar correctamente las acciones de los otros. No obstante, ello no haría justicia a la complejidad del caso y se perderían características importantes de la interacción (especialmente su carácter cíclico, fruto de un proceso de retroalimentación).

29. Putnam desarrolló el concepto de juego de dos niveles para analizar las relaciones entre la arena política internacional y la arena política doméstica.

30. La Verdad, 21/2/1988. 
En el caso de un juego de varios niveles como el que se ha propuesto en este artículo, sin embargo, la presunción de conocimiento común se restringe a cada uno de los juegos (es decir, se presume que cada jugador decide atendiendo al conocimiento de las características del juego que en ese momento está jugando) y las consecuencias inintencionadas, por tanto, son fruto de la combinación de diversas estrategias emprendidas en distintas mesas negociadoras y resultan, por ello mismo, difícilmente previsibles ${ }^{31}$ para los actores.

\section{¿Un modelo generalizable?}

A pesar de la particularidad del conflicto estudiado, cabe preguntarse qué rasgos tiene que sean susceptibles de generalización y apuntar hacia un posible modelo que pudiera emplearse para estudiar otros casos. Haciendo abstracción del contenido empírico concreto, pensemos en la relación entre dos partes en conflicto $(\mathrm{A}$ y $\mathrm{B})$ y un mediador, tal que:

1. A y B prefieren, en primer lugar, ser las únicas en negociar con el mediador y, en último lugar, ser las únicas en no negociar con el mediador.

2. A y B prefieren que el mediador se comprometa a satisfacer sus demandas a que no se comprometa. En el primer caso, prefieren no movilizarse a movilizarse, y en el segundo, prefieren movilizarse a no movilizarse.

3. El mediador es más sensible a las demandas de uno de los dos (digamos A), pero no es absolutamente insensible a las demandas del otro (digamos B). En cualquier caso, prefiere que los colectivos no se movilicen a que sí lo hagan.

Estas hipótesis sobre las motivaciones plausiblemente pueden conducir, como en el caso de la crisis de la comarca minera de Cartagena-La Unión, a un tipo de proceso caracterizado por la aparición de efectos perversos. Dicho proceso, además, mostraría muchos de los rasgos de la formalización recientemente propuesta por Cristiano (2001: 164) ${ }^{32}$ :

a) Ninguno de los participantes se propuso, a nivel de conciencia discursiva, producir el resultado.

b) Ninguno conoce, a nivel de conciencia discursiva, los mecanismos específicos de su producción.

c) Todos los participantes tienen «razones» (a nivel de conciencia discursiva, conciencia práctica) o motivaciones para actuar como lo hacen.

d) Cada uno aporta, en proporción diversa según sus recursos, a la producción del resultado.

e) El resultado es opuesto a lo pretendido (a nivel de «conciencia práctica» o «discursiva») por al menos una parte de los participantes.

31. Hemos tratado esta cuestión con mayor profundidad en Linares (2003).

32. Se ha excluido de la lista de Cristiano las afirmaciones de que ninguno conoce «el resultado que se produce» $\mathrm{y}$ «ninguno puede, con el sólo expediente de abstenerse de actuar, impedir la producción del resultado». 
f) El proceso no tiene la forma lineal y cerrada de un conjunto de acciones seguidas por un resultado; es el resultado progresivo de diversas acciones, cada una de las cuales ratifica y fortalece la dirección del proceso.

g) Las acciones de los participantes tienen un grado importante de fijeza e institucionalidad, por lo cual,

h) La evitación del efecto implica alterar, junto con las acciones, algunos de los intereses.

\section{Postescripto para curiosos: ¿cómo finalizó la crisis de la minería de la comarca de Cartagena-La Unión?}

La incógnita del comprador de Peñarroya se despejó en septiembre de 1988 con el siguiente comunicado:

La Sociedad Minera y Metalúrgica de Peñarroya-España, titular de las minas, empeñada en garantizar el empleo de sus trabajadores, después de realizar estudios profundos de la situación, ha llegado a la convicción de que ello exige, además de mantener la mina en explotación el tiempo posible, promover en otros sectores actividades complementarias capaces de generar puestos de trabajo. Tras estudiar las posibles opciones de compra, Peñarroya-España ha llegado a un acuerdo con Portmán S.A. [...] que adquiriendo la totalidad de los elementos patrimoniales de las minas y en perfecta sincronización con la actividad minera, desarrollará los negocios alternativos referidos ${ }^{33}$.

Tales negocios alternativos no eran otros que los de la construcción, aprovechando el amplísimo coto minero, susceptible de explotación turística, vendido por Peñarroya-España. El mantenimiento de los puestos de trabajo, no obstante, seguía dependiendo de la posibilidad de explotar Los Blancos III. Tras diversas vicisitudes difícilmente resumibles (Linares, 2000, 2001), nunca se llevaron a efecto los proyectos urbanísticos y, en cuanto a la actividad minera, a pesar de construirse un nuevo lavadero que evitaba los vertidos al mar, se le puso punto y final en 1991 con el despido de 303 trabajadores.

\section{Bibliografía}

BAERT, P. (1991). «Unintended consequences: a typology and examples». International Sociology, vol. 6, n. ${ }^{\circ}$ 2, p. 201-210.

Becker, G.S. (1976). The Economic Approach to Human Behavior. Chicago: The University of Chicago Press.

Cristiano, J. (2001). «Males involuntarios. Para una reapropiación del concepto de efectos perversos». Papers, 65, p. 149-166.

ElSTER, J. (1994). Lógica y sociedad. Contradicciones y mundos posibles. Barcelona: Gedisa. 
Fernández Valbuena, S. (1989). «La industria en la Región de Murcia». Papeles de Economía Española. Economía de las Comunidades Autónomas, vol. 8, p. 256-278.

Gallego RodrígueZ, A.; Monllor DomíngueZ, J. (1993). «Recursos naturales: la minería». En Colino, J. (dir.) (1993). Estructura económica de la Región de Murcia. Madrid: Cívitas.

Gea Javaloy, R. (1986a). "El sector del plomo en España». Papeles de Economía Española, vol. 29, p. 271-281.

- (1986b). "El sector del zinc». Papeles de Economía Española, vol. 29, p. 321-331.

GiBBOns, R. (1993). Un primer curso de teoría de juegos. Barcelona: Antoni Bosch.

Linares MarTínez, F. (2000). La crisis de la comarca minera de Cartagena-La Unión (1987-1991). Un análisis sociológico fundamentado en el paradigma de la elección racional. Universidad de Murcia. Tesis doctoral.

- (2001). La crisis de la comarca minera de Cartagena-La Unión (1987-1991). Un análisis sociológico sobre las paradojas de la acción racional. Cartagena: Ayuntamiento de Cartagena.

- (2003). «Weak and Strong Unintended Consequences». Comunicación presentada al $6^{\text {th }}$ Conference of the European Sociological Association, Murcia, del 23 al 26 de septiembre. Puede consultarse el texto íntegro en: www.kuleuven.ac.be/social theoryeurope/STESA-03/Francisco_Linares.pdf

Martín Camino, M. (1996). «Del final de la Edad de Bronce al Mundo Bizantino». En Tornel Cobacho, C. (coord.) (1996). Manual de Historia de Cartagena. Murcia: Universidad de Murcia.

MERTON, R.K. (1936). «The unanticipated consequences of purposive social action». American Sociological Review, vol. 1, p. 894-904.

Peñarroya-España (1987a). Proyecto de la corta Los Blancos III. La Unión.

- (1987b). Plan Estratégico de Adaptación Competitiva. La Unión.

PuTNAM, R.D. (1988). «Diplomacy and domestic politics: the logic of two-level games». International Organization, vol. 42, n. ${ }^{\circ}$ 3, p. 427-460.

SEGURA, J. (coord.). 1989. La industria española en la crisis. Madrid: Alianza.

Urrea Pagán, A. (1992). Historia de Llano del Beal (2 tomos). Cartagena: Asociación de Vecinos de Llano del Beal.

VilaR, J. B.; EgEa Bruno, P.M. (1985). La minería murciana contemporánea (18401930). Murcia: Universidad de Murcia.

Vilar, J.B.; Egea Bruno, P.M.; FernándeZ Gutiérrez, J.C. (1990). La minería murciana contemporánea (1930-1985). Madrid: Instituto Tecnológico GeoMinero de España. 